\title{
Beam loss Monitors for FEL using optical Fiber
}

\author{
F. Wulf, M. Körfer, W. Goettmann, H.-J. Grabosch
}

\begin{abstract}
Beam losses and beam profiles at particle accelerators can be determined by measuring the ionizing radiation outside the vacuum chamber. The next generations of free electron lasers require novel solutions for beam loss monitor and radiation detection systems. A new concept at the Free-ElectronLaser Hamburg (FLASH) and Photo Injector Test Facility Zeuthen (PITZ) was developed and put into operation. The concept based on optical fibers used as radiation detection sensor. Slow beam loss monitors determine the total ionization dose at selected positions or along the beam line and undulator sections in a response time of a few milliseconds by measuring the radiation-induced attenuation of the fiber. Fast beam loss monitors detect the Cerenkov light generated by relativistic electrons or gamma rays penetrating radiation hard fiber. The new beam loss position and beam profile monitor systems utilize this effect. The time response is below milliseconds with a time resolution of some nanoseconds. These monitors provide a technique to improve the beam performance and trace the stability of the over all setting parameters. This paper presents the new diagnostic methods and the operation experience of the systems with a focus on the fast beam loss monitor systems.
\end{abstract}

\section{INTRODUCTION}

B EAM loss monitor systems (BLM systems) are an essential part of linear accelerators and storage rings. They allow the understanding of the beam loss mechanisms during commissioning and operation and provide an option for an emergency shutdown. Well-known beam loss systems [1], like a) long and short ionization chamber, b) combination of scintillator and photomultiplier and c) PIN photo-diodes, d) scintillation counter, e) electron multipliers, f) cryogenic calorimeters have still some deficiency [2]- [7]. They do not cover the complete section of the accelerator, especially at large undulator systems. The position and time resolution is insufficient in some applications. New BLM systems devel-

Manuscript received

Dr. Friedrich Wulf is with the Helmholtz-Zentrum Berlin für Materialien und Energie (HZB), Lise-Meitner Campus, Berlin, D-14109, Germany (telephone: +4930 80622379; fax: +4930 80622096, e-mail: wulf@helmholtzberlin.de).

Dr. Markus Körfer is with the Deutsches Elektronen-Synchrotron Ein Forschungszentrum der Helmholtz-Gemeinschaft, (DESY), Hamburg, D-22607, Germany (telephone: +4940 8998 3714; fax: +4840 8998 4305; e-mail: markus.koerfer@desy.de)

Walter Goettmann was with the Helmholtz-Zentrum Berlin für Materialien und Energie (HZB), Lise-Meitner Campus. $\mathrm{He}$ is now retired. (e-mail: wgoettmann@t-online.de)

Dr. Hans-Jürgen Grabosch is with the Deutsches Elektronen-Synchrotron Ein Forschungszentrum der Helmholtz-Gemeinschaft, (DESY), Zeuthen, D-15738 Germany, (telephone: +49033762 77347; fax: +493376277330); e-mail: hans-juergen.grabosch@desy.de)

This work was partially support by the Impuls- and Vernetzungsfonds der Helmholtz-Gemeinschaft, Germany, No. VH-FZ-006. oped particularly for FLASH overcame these problems. They are tested and approved during the operation at FLASH and at the Photo Injector Test Facility (PITZ), DESY Zeuthen. The have been tested also during real operation at the following accelerator facilities: MAX-LAB [8], BESSY II, DELTA [ 9]-[10], SLS.

\section{Classification of BeAm Loss Monitor Systems}

A S shown in Fig. 1 the new BLM concept distinguished two categories. One system measure the total ionizing dose (TID) generated by the beam losses as a function of radiation induced attenuation (RIA). With an optical power meter, the RIA measured at fixed position along the beam line, particularly at the undulator. The resolution of the absorbed dose of the so-called local sensor system is about 60 mGy. The updating time is - depending of the number of used sensors - in the range of milliseconds. The sensor fiber saturates at about $2 \mathrm{kGy}$ and must than be replaced. In high dose environment, Fiber Bragg Gratings can be used as local sensor system by measuring the Bragg wavelength shift (BWS) as a function of the absorbed dose [11]. The influences of the production process of the fiber it self leads to a factor of three in BWS. A variation of the process parameter provides a BWS difference of nearly a factor of ten [12]. Up to a dose level of $100 \mathrm{kGy}$ the fiber show no saturation and is almost independent of the radiation dose rate. They can be used up to a level of 1 MGy [12].

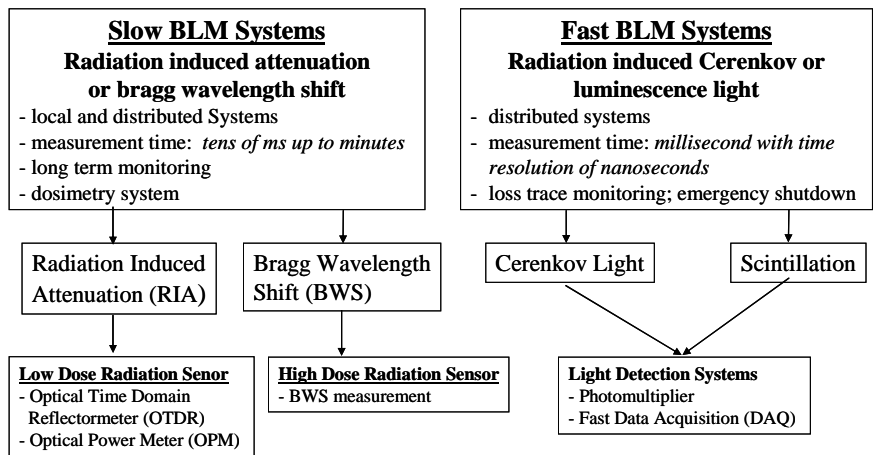

Fig. 1. Classification flow chart of beam loss monitors systems using optical fiber sensor.

The second set up is a distributed sensor system that makes use of Optical Time Domain Reflectormeter (OTDR) measurements. As result of the attenuation measurement, the position and TID along the beam line or undulators is calculated. At FLASH, the typical sensor length is less then $100 \mathrm{~m}$. The update time is in the range of some seconds up to 
minutes depending of the dose and position resolution, which is about $3 \mathrm{~Gy}$ and $1.5 \mathrm{~m}$, respectively. These systems are explained in more detail in references [13]-[15].

The fast beam loss monitor system detects the generated Cerenkov light in the fiber. The typical used fiber length at FLASH is about $40 \mathrm{~m}$. Under this condition, a position resolution of $25 \mathrm{~cm}$ with a measurement time below millisecond can be achieved. This makes the system also applicable for emergency shutdown. The time resolution of the measured pulses is one nanosecond. Please note: scintillation fibers are usable as fast radiation sensors, but they are very radiation sensitive. In addition, the initial light attenuation is high and therefore they are not applicable as beam loss monitor over long distance or in applications with high dose environment.

\section{OPtimizATION AND SELECTION OF OPTICAL FIBERS}

SINCE more than 30 years, it is well known that optical $\checkmark$ fibers can be used as radiation sensor. The nonsatisfying applications in the past rely at most on insufficient knowledge and control of the producing process of the fibers. An individual characterization of each lot is indispensable. This is a key function for further usage of fiber as radiation sensor. The influence of core material, cladding thickness, drawing speed of the fiber and coating material of pure silica core stepindex fibers have been investigated in detail [16]. Fast BLM require radiation resistant fiber with high bandwidth and large core diameter. Experience at the Fraunhofer Institut Naturwissenschaftlich-Technische Trendanalysen INT, Euskirchen and Hahn-Meitner-Institut Berlin (now Helmholtz-Zentrum Berlin für Materialien und Energie), Germany, in optical fiber characterization facilitate the collaboration to an intensive selection process. The selected fiber for the fast beam loss monitor systems is a fiber with a pure silica core and high $\mathrm{OH}$-content. This type of fiber is in general radiation resistant. However, F-doped fiber can reach higher radiation level without increasing the RIA [17]. The presented BLM systems use a multi-mode step-index fiber with a core diameter of 300 $\mu \mathrm{m}$. The core diameter is a good compromise between the irradiation sensitivity and the required bandwidth. A black nylon buffer shields the fiber against the ambient light. The total diameter of the fiber is only $900 \mu \mathrm{m}$.

\section{FAST BEAM LOSS MONITOR SySTEMS (CERENKOV LIGHT)}

$M^{\prime}$ ANY previous publications describe the measurement of ionizing radiation by detection of the generated Cerenkov light in optical fibers. The implementation and systematic optimization of this technology was missing. At FLASH two measurement systems are installed. The measurement set up for the undulator section and upstream beam line section is shown in Fig. 2. At the undulators, four fibers are grouped together in equidistant radial distance around the vacuum chamber sharing the same cut outs with the OTDR fiber (Fig. 3). The fibers are connected inside the tunnel to photomultipliers (PMT). The PMTs are carefully selected with respect to their sensitivity. Low noise preamplifier amplifies the signals, which are than digitized by fast ADC (1 GS/s, $1 \mathrm{GHz}$ bandwidth). The measurement system is installed outside the radiation area of the tunnel in a PXI-System. The system is synchronized with the bunch clock and controlled by an embedded CPU. The system is integrated in the FLASH control system. Selected events can be logged together with the machine status. Maintenance is executed via remote control from the HMI. This system is used as beam loss position monitor (BLPM) as well as beam profile monitor (BPM).

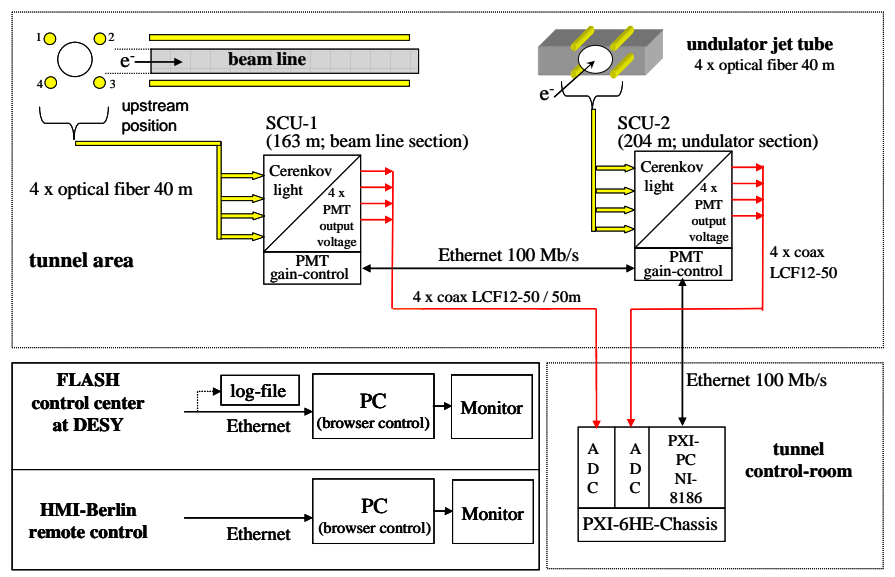

Fig. 2. Measurement set up for BLPM and BPM at FLASH. One sensor group controls the undulator and a second one the beam line.

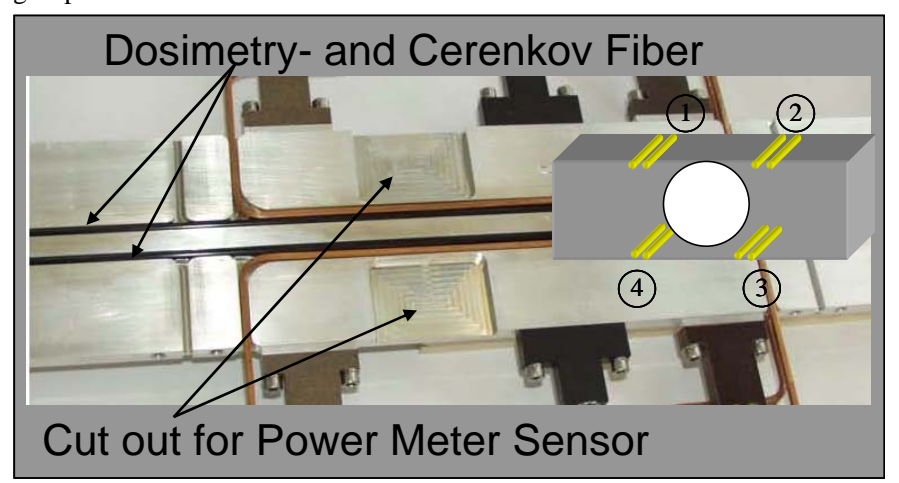

Fig. 3. Detail of the vacuum chamber line of the undulator show the cut out for the power meter sensor, the OTDR and Cerenkov fiber

\section{A. Fast Beam Loss Monitor}

Fast BLM require radiation resistant fiber with high bandwidth. Electrons with energies above $175 \mathrm{keV}$ generate Cerenkov light in fibers $(n=1.46)$. Detailed information about the Cherenkov effect in optical fibers can be found in [18][23]. Based on this effect, powerful BLM systems like beam loss position monitor (BLPM) [24] and beam profile monitor (BPM) [25] were developed.

Typical curves of the Cerenkov light generated in the optical fibers over a length of six undulators during routine operation at FLASH are shown in Fig. 4.

\section{B. Beam Loss Position Monitor}

The position of the radiation source is calculated by the time difference between a maximum peak height of the light 
pulse and the bunch clock trigger. The accurate location of the secondary shower with respect to the beam line layout can be determined by vacuum components like optical transition radiation (OTR) screen or wire scanner with their know position. It has been shown, that the position can be determined with an accuracy of about $25 \mathrm{~cm}$ measured at PITZ [26]. Due to the fact, that the velocity of relativistic electrons in vacuum $(\sim \mathrm{c})$ is higher than the light velocity in the

fiber

$(\sim 0.66 \cdot c)$, the measured light signals are expanded or compressed depending on the position of the PMTs relative to the beam line. If the PMTs are installed at the upstream position, the measured light signals are stretched by a factor of 2.5 relatively to the electrons in the vacuum chamber. Installing the PMTs at the downstream position, the signals are compressed by a factor of two. This gives the possibility to detect single bunches at the downstream position with five times higher repetition rate as at the upstream position. However, with the same bandwidth and sample rate the position resolution is than five times lower [27]. In Fig. 5 the principle result of BLPM measurement is shown. The losses are generated by an inserted OTR-screen. The intensity of the

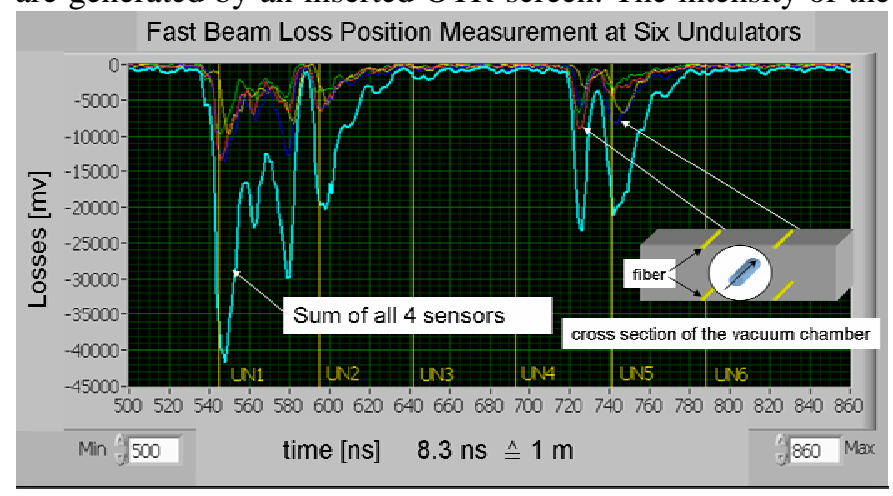

Fig. 4. Screen shot at FLASH, in March 2009. At six undulators, the Bremsstrahlung level at four positions is shown. An adjustable persistence mode improves the quality of the visualisation.

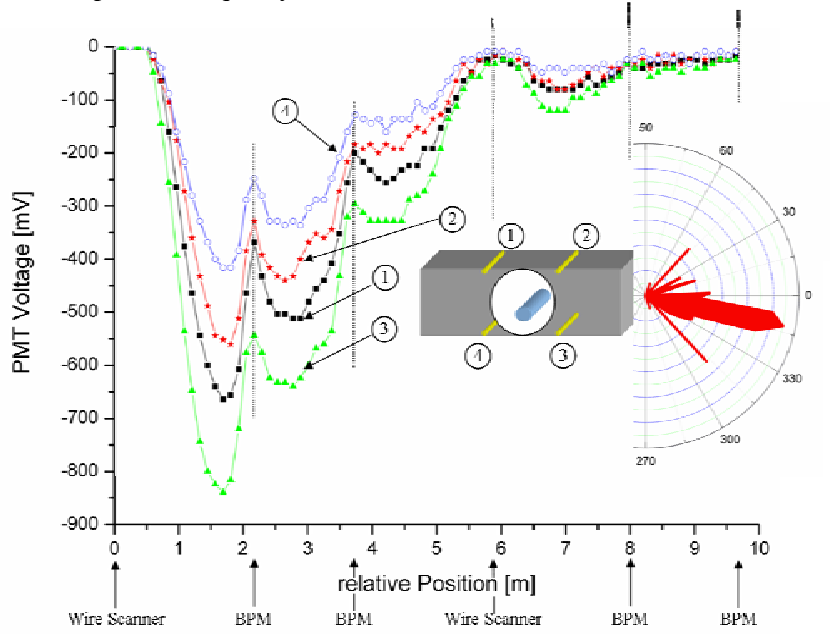

Fig. 5. Typical results of a BLPM measurement measured at the undulator section at FLASH. The middle inset shows the position of the fibers at the vacuum tube. The right inset shows the calculated centroid of the Bremsstrahlung in lateral direction of the beam line (downstream view).

loss shower increases at the first quadrupole. The local minima of the signal are caused by the shielding effect of the components (quadrupoles, vacuum pumps e.g.) and transversal distance variation of the fiber with respect to the beam line. Nevertheless, using the maximum values of the signals, an envelope function can be calculated to define the direction of the centroid of the Bremsstrahlung shower as shown in the inset of Fig. 5. The generated Cerenkov light in optical fibers depends on a) the velocity of the particle, b) the incident angle, c) the energy of the particle, d) the distance between the particle trajectory and the center of the fiber. In addition, the light measured at the one end of the fiber is convolved with the a) the fiber core diameter, b) the refraction index, c) spectral attenuation of the fiber and, d) the spectral quantum efficiency of the light detector. The maximum generated light in the fiber can be achieved when the incident angle between the particle trajectory and the fiber is about $45^{\circ}$ [18]-[23].

Using the fiber in a BLM system the optimum measurement condition can not always be fulfilled. It is therefore necessary to define the sensitivity of the individual BLM system.

Fig. 6 shows the integral light generated of the BLM system installed at PITZ as a function of the charge of one bunch. The used electron energy is $12 \mathrm{MeV}$ and as target, a tungsten slit mask is used. The result shows a good linearity between the measured light and the primary electron charge. Between 300$700 \mathrm{pC}$, the beam was not in the centre of the target and this caused a small parallel shift of the curve. Therefore, a measurement of the absolute value of the primary loss electrons is not possible, but for each set up the resolution can be determined. In this case, the resolution is $0.2 \mathrm{pC}$. Novel pre-amplifier increases the sensitivity of the BLM system by a factor of 10 .

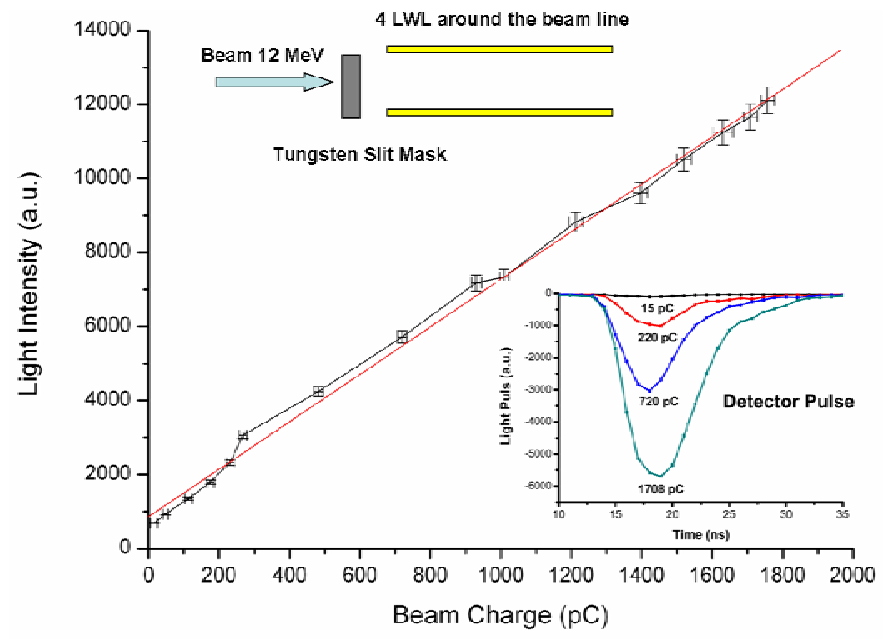


Fig. 6. light intensity as a function of the beam charge measured at PITZ inject

\section{Beam Profile Monitor}

Beam profile measurement is an essential part of the beam diagnostic. Conventional systems use wire scanner systems (WS) [28] where only a fraction of the generated Bremsstrahlung in longitudinally direction is measured by a PMT at the end of the interesting beam line section. The efficiency of this measurement principle is additionally reduced due to the shielding effect of the beam line components. In contrast, the build in BLPM can be used to measure the Bremsstrahlung in the longitudinal as well as in the transversal direction, particularly direct after the wire scanner. The interesting parts are the beam core and tails. The measurement of the beam profile requires a large dynamic range of the detection system. In addition, parallel to the installed Cerenkov fiber group a second set of fibers are used. This gives the opportunity to measure at the same time with low and high amplification ranges of the PMT. This is necessary, because the sensitivity of the PMT after high illumination level needs a too long recovery time with respect to the fast movement of the wire scanner itself. Figure 7 shows the good correlation between measurements with standard WS systems and the new BPM system. Detailed experimental results are given in [25]. As explained before, a new pre-amplifier increases the sensitivity of the BPM system by a factor of 10 . This offers the possibility to measure the beam tail with higher resolution.

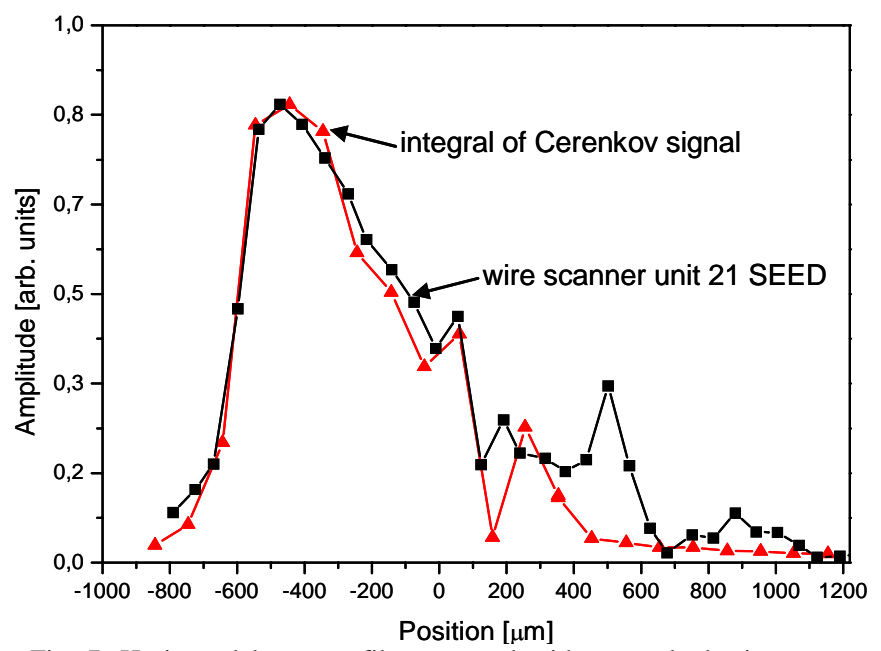

Fig. 7. Horizontal beam profile measured with a standard wire scanner system at FLASH, compared to results obtained with an optical fiber BPM system. The maximum values are normalized. The measurements were taken one after another.

\section{SUMMARY}

$\mathrm{A}$ T FLASH and PITZ it was shown, that fiber sensors used in slow beam loss monitor systems as well as in fast BLM systems are useful tools for the optimization and control process of particle accelerators. In general, fiber sensor can be used in nearly inaccessible gaps or regions with high electrical or electromagnetic fields. It is an indispensable precondition, that a careful selection of the sensor fiber is necessary to meet the calibration requirements. The local dosimeter system can be placed anywhere with individually adapted sensitivity and detection area. These sensors measure local doses with a resolution of some tens of milligrays in real time. Distributed dosimeter use the same fiber type. The fibers are installed up to several hundred meters along the accelerator beam line, tunnel or undulators, measuring the total ionization dose (TID) down to a few Gray with a position resolution of about $1.5 \mathrm{~m}$. The measurement time is in a range of some minutes, depending of the required dose accuracy. The system is applicable to supervising the radiation level of the total accelerator environment up to a maximum dose level of 2-3 kGy.

Fast BLM systems like BLPM and BPM enable the operator to control the beam loss along the entire beam line in transversal and longitudinal dimensions. It allows the identification of single bunches within one bunch train. The system response is in the range of millisecond with a time resolution of nanosecond and a position resolution of about 25 $\mathrm{cm}$. This allows an interactive control of the beam optimization process and gives the opportunity to trace the stability over all setting parameter of the beam and the accelerator parameter drift, respectability. At PITZ, the BLPM will be used as trigger for the fast emergency shut down system. A careful selection of the radiation resistance fiber is an indispensable prediction for reliable operation. Optimized BPM systems provide a beam profile measurement with higher sensitivity to the beam core and beam tail compared to standard wire scanner systems. The important parameters of BLM systems are summarized in table 1 . 


\begin{tabular}{|c|c|c|c|c|}
\hline \multirow[b]{2}{*}{ Application } & \multicolumn{3}{|c|}{ Slow BLM Systems } & \multirow{2}{*}{$\begin{array}{c}\text { Fast BLM Systems } \\
\begin{array}{c}\text { Beam Loss Position Monitor } \\
\text { and }\end{array} \\
\text { Beam Profile Monitor }\end{array}$} \\
\hline & $\begin{array}{l}\text { Distributed Dosimeter } \\
\text { System }\end{array}$ & $\begin{array}{l}\text { Local Dosimeter } \\
\text { System }\end{array}$ & $\begin{array}{c}\text { Local Dosimeter } \\
\text { System (High Dose) }\end{array}$ & \\
\hline Measurement principle: & $\begin{array}{c}\text { Optical Time Domain } \\
\text { Reflectormeter }\end{array}$ & Optical Power Meter & $\begin{array}{l}\text { Bragg Wavelength } \\
\text { shifting ( } \Delta \text { BWS) }\end{array}$ & Cerenkov Light \\
\hline $\begin{array}{l}\text { Measurement time } \\
\text { (detection response) }\end{array}$ & minutes & ms to minutes & ms to sec & $\leq \mathrm{ms}$ with time resolution of $1 \mathrm{~ns}$ \\
\hline $\begin{array}{l}\text { Range of maximum dose } \\
\text { TID [Gy] }\end{array}$ & $\begin{array}{c}3-450 \\
\text { limited by OTDR }\end{array}$ & $\begin{array}{l}\text { 0.06- } 2000 \\
\text { limited by fiber type }\end{array}$ & $\begin{array}{l}2 * 10^{3}-10^{6} \\
\text { limited by fiber type }\end{array}$ & $\begin{array}{l}\text { only a rough estimation possible, } \\
\text { fiber can used until } 1 * 10^{5}\end{array}$ \\
\hline Wavelength range & $850-1330 \mathrm{~nm}$ & $860 \mathrm{~nm}$ & $\begin{array}{c}820 \mathrm{~nm}-1,55 \mu \mathrm{m} \\
\Delta \lambda_{\mathrm{B}}=5-350 \mathrm{pm}\end{array}$ & $200-850 \mathrm{~nm}$ \\
\hline
\end{tabular}

* Depending on the maximum dose and required position resolution

\section{ACKNOWLEDGMENT}

We thank Dr. H. Henschel, Dr. J. Kuhnhenn, U. Weinand, O. Köhn, of the Fraunhofer Institut für Naturwissenschaft-lich-Trendanalysen, INT, Euskirchen, Germany, for the strong engagement over many years developing the powerful measurement set ups, data acquisition systems, the optimization and selection process of optical fibers and valuable contributions to the successful execution of this challenging research project. We thank M. Sachwitz of DESY Zeuthen and R. Awwad, of DESY for supporting the measurements.

\section{REFERENCES}

[1] K. Wittenburg, "Beam loss monitoring and control", Proceedings of the 8th EPAC 2002, Paris, France, pp 109-113.

[2] K.Wittenburg, "Beam loss detection", Proc 1st DIPAC93 CERN PS/93-35 (DB) pp11-20.

[3] B.Joly, U.Weinrich, G.A.Naylor, "Beam loss monitors at the ESRF", Proc. DIPAC 1999, Chester, UK, IT 3, pp 3-6.

[4] H.Burkhardt, "How to use beam loss monitors at the LHC?" Proc. Chamonix XI Workshop, Jan. 2001, CERN-SL-2001-003 DI.

[5] P. Michel, J. Teichert, R. Schurig, H. Langenhagen, "Beam loss detection at radiation source ELBE", Proc. DIPAC 2003, CT05, pp 65-67.

[6] J. Roelfe et al., "Long ion chambers systems for the SLC", SLACPUB-4925, 3/1989.

[7] K. Wittenburg, "Beam loss detection" DESY; Hamburg, Proc. 1st European Workshop on Beam Diagnostics and Instrumentation for Particle Accelerators, Montreux, Switzerland, 3-5.5.1993,CERN PS/93-35 (BD).

[8] J. Bahrdt, et al., "Status of the undulator system on the seeded HGHG-FEL test bench at MAX-lab", International Free Electron Laser Conference 2007, Novosibirsk (2007).

[9] G. Schmidt, E. Kasl, K. Wille, M. Körfer, H. Henschel, J. Kuhnhenn, "Optical Fibre Beam Loss Monitors for Storage Rings at DELTA", Proc. EPAC 2002, Paris, France, pp 1969-1971.

[10] F. Rüdiger, G. Schmidt, K. Wille, M. Körfer, W. Göttmann,"Beam Loss Position Monitoring with optical Fibres at DELTA", Proc. EPAC June 23- 27, 2008, Genua, Italy, TUOCM03, p 1032.

[11] K. Krebber, H. Henschel, U. Weinand, "Fibre Bragg gratings as high dose radiation sensors?", Measurement Science and Technology, Volume 17, Issue 5, pp. 1095-1102 (2006).
[12] Henning Henschel, Stefan K Hoeffgen, Katerina Krebber, Jochen Kuhnhenn, and Udo Weinand, "Influence of Fiber Composition and Grating Fabrication and the Radiation Sensitivity of Fiber Bragg Gratings", IEEE Transaction on Nuclear Science, Vol. 55, No. 4, August 2008, pp 2235-2242.

[13] H. Henschel, M Körfer, J. Kuhnhenn. U. Weinand, F. Wulf., "Fibre optic radiation sensor systems for particle accelerators", Nuclear Instruments and Methods in Physics Research Section A: Accelerators, Spectrometers, Detectors and Associated Equipment, 2004. 526(3): p. 537-550.

[14] H. Henschel, M. Körfer, J. Kuhnhenn, U. Weinand, F. Wulf, "Fibre optic sensor solutions for particle accelerators", Proc. 17th International Conference on Optical Fibre Sensors, 23-27 May 2005, Bruges, Belgium, pp 515-518.

[15] F. Wulf, M. Körfer, "Local beam loss and beam profile monitoring with optical fibers", Proc. DIPAC 2009, 25-27 May 2009, Basel, Switzerland, WEOA01, pp 411-417.

[16] Kuhnhenn, J. and H. Henschel, "Influence of coating material, cladding thickness, and core material on the radiation sensitivity of pure silica core step-index fibers", 8th European Conference on Radiation and Its Effects on Components and Systems (RADECS 2005), Cap d'Agde, France.

[17] Thijs Wijnands, Luit Kort De Jonge, Jochen Kuhnhenn, Stefan Klaus Hoeffgen, and Udo Weinand, "Optical Absorption in commercial Single Mode Optical Fibers in a High Energy Physics Radiation Field", IEEE Transactions on Nuclear Science, Vol.55 (2008), No.4, pp.2216-2222.

[18] G. Anzivino et al. "Quartz fibre calorimetry- Monte Carlo simulation", Nuclear Instruments and Methods in Physics Research A 357, 1995, pp 380-385.

[19] G. Anzivino et al. "Recent developments in quartz fibre calorimetry", Nuclear Instruments and Methods in Physics Research A 357, 1995, pp 369-379.

[20] K. Arrington et al. "Cerenkov fiber sampling calorimeters", IEEE Transactions on Nuclear Science (ISSN 0018-8499), vol. 41 no. 4, pt.1, pp 840-844.

[21] Wang, Yao-Cai, Shi, Yi-Wie, Jiang Hong-Tao, "Passive optical fibre sensor based on Cerenkov effect", Proc. SPIE vol. 1572, International Converence on Optical Fibre Sensors in China, 1991, pp 32-37.

[22] B. L. Pruett et al., "Gamma-Ray to Cerenkov-light conversion efficiency for pure-silica-core optical fibers", Proc. SPIE vol. 506, Fiber Optics in Adverse Environments II, 1984, pp 10-16.

[23] W. Schneider, U. Babst, O.A. Balk, E. Hochhäuser, "Radiationinduced light emission in silica core fibers", Proc. SPIE vol. 506, Fiber Optics in Adverse Environments II, 1984, pp 189-195.

[24] W. Goettmann, F. Wulf, M. Körfer, J. Kuhnhenn, "Beam Loss Position Monitor using Cerenkov Radiation in Optical Fibers", Proc. of DIPAC 2005, Lyon, France, POW026, pp 301-303. 
[25] W. Goettmann, M. Körfer, F. Wulf,"Beam Profile Measurement with Optical Fiber Sensors at FLASH", Proc. of DIPAC 2007, Vience, Italy, TUPB 25, pp 123-125.

[26] H.-J. Grabosch, W. Goettmann, "Results of Test Measurements, Simulation of Beam-Loss with FLUKA", SEI Tagung, Report; HMIB 619, ISSN 0936-0891, Ruhr Universität Bochum, 24. - 26. Sept. 2007, pp 176-191.

[27] W. Goettmann, et. al., "Strahlverlustmessung durch Messung der Cerenkovstrahlung in Lichtwellenleitern" SEI Herbststagung, 26. 28. Sept. 2005 Berlin, HMI-B 2005, ISSN 0936-0891, pp 49-61.

[28] N. von Bargen et al., "Prototype of a Wire Scanner for TTF II", TESLA Report 2002-8. 